\title{
Post-mortem thermal angiography: a pilot study on swine coronary circulation
}

\author{
Paolo Fais $^{1}$ - Maria Carla Mazzotti ${ }^{1}$. Massimo Montisci ${ }^{2} \cdot$ Chiara Palazzo $^{1} \cdot$ Ornella Leone $^{3} \cdot$ Giovanni Cecchetto $^{2}$. \\ Guido Viel $^{2}$ (D) Susi Pelotti ${ }^{1}$
}

Received: 31 January 2018 / Accepted: 7 September 2018 / Published online: 14 September 2018

(C) Springer-Verlag GmbH Germany, part of Springer Nature 2018

\begin{abstract}
Thermal imaging (TI) allows the detection of thermal patterns emitted from objects as a function of their temperature in the longinfrared spectrum and produces visible images displaying temperature differences. The aim of this pilot study was to test TI to visualize the coronary circulation of swine hearts. Thirty swine hearts were prepared for ex situ coronarography, and thermal images were acquired through a FlirOne thermal camera (FLIR Systems ${ }^{\circledR}$ ) paired with a Google Android Smartphone. Coronary arteries were cannulated, namely the anterior interventricular artery, the circumflex branch of the left coronary artery, and the right coronary artery. The heart was cooled, and contrast medium (CM) consisting of distilled water heated to $40{ }^{\circ} \mathrm{C}$ was injected in a coronary vessel, while thermal images were captured. These steps were repeated for each coronary vessel and under experimentally simulated coronary heart disease. Thermal imaging coronarography (TIC) allowed a clear representation of the morphology and course of the coronary vessels and of experimentally simulated coronary heart disease, moreover, demonstrated to be easy to perform during or after autopsies on ex situ hearts, non-destructive, reproducible, and cheap. On the basis of these preliminary results, TIC might allow a subsequent more focused and comprehensive cardiopathological examination of the heart, which remains mandatory for the definitive diagnosis of coronary heart disease. Although these preliminary results seem encouraging, further systematic studies on human hearts, both normal and pathological, are necessary for estimating the sensitivity and specificity of the proposed method and to draw any definitive conclusion.
\end{abstract}

Keywords Thermal imaging $\cdot$ Post-mortem coronarography $\cdot$ Cardiopathology $\cdot$ Post-mortem imaging $\cdot$ Ex situ coronarography

\section{Highlights}

- Thermal imaging allows the clear imaging of swine coronary vessels.

- Thermal imaging recognizes simulated occlusion/sub-occlusion of coronary arteries.

- Thermal imaging coronarography (TIC) is cheap and easy to perform.

- TIC might be performed during the autopsy or even later and in a different setting.

- TIC is reproducible and non-destructive.

Guido Viel

guido.viel@unipd.it

1 DIMEC, Department of Medical and Surgical Sciences, University of Bologna, 40126 Bologna, Italy

2 Legal Medicine and Toxicology, University Hospital of Padova, Via Falloppio, 50, 35121 Padova, Italy

3 Department of Pathology, Sant'Orsola-Malpighi University Hospital, Bologna, Italy

\section{Introduction}

Thermal imaging (TI), also known as infrared imaging, is a technique originally developed for military use that shows the thermal patterns emitted from objects in the long-infrared spectrum, invisible to the human eye [1]. All objects emit infrared energy (heat) as a function of their temperature: the hotter the object, the more infrared radiation emitted. Thermal cameras, also known as infrared cameras, enable the conversion of invisible infrared radiation into visible images and can be used to visualize temperature differences [2].

TI has been proposed in different clinical settings, such as to estimate the blood perfusion of skin and tissues related to the metabolic activity [3] and a number of different pathologies [4]. In particular, the more relevant applications of TI published in the medical literature were the following: the diagnosis and screening of breast cancer [5-8], the assessment of burns [9, $10]$, the study of inflammation and the related therapy [11-13], muscular pathology [14, 15], fever in the screening 
of SARS infection in airports [16-18], angiogenesis [19], and cardiac surgery [20]. More recently, TI applications have been proposed for forensic purposes on post-mortem samples. TI has been used in studies dealing with the post-mortem interval to estimate the supravital energy production in early post-mortem phase [21] or related to forensic entomology. In particular, TI was used to investigate the temperature of exposure of insects colonizing the carcasses [22] and to detect cadaveric remains colonized by insects and larval masses producing heat [23, 24]. On the other hand, to the best of our knowledge, TI has not been investigated yet for the imaging of vascular structures on postmortem samples. The aim of this pilot study was to test TI to visualize the coronary circulation of ex situ swine hearts. Imaging of the coronaries was acquired also simulating experimentally coronary artery occlusion and/or sub-occlusion. Results have been discussed focusing on the pros and cons of thermal imaging coronarography (TIC), pointing out any potential future perspectives of TI in post-mortem angiography.

\section{Materials and methods}

TIC was performed on 30 hearts of 18-month-old swines, which were obtained from a local butcher and prepared for ex situ coronarography.

\section{Preparation of the heart-cannulation of coronary arteries}

For each heart, according to C. Bruguier et al. [25], using blunt dissection, the root of the aorta and the pulmonary artery were separated down to their proximal portion. A 2-cm-long portion of the aorta was removed with a transversal cut in order to identify the three aortic cusps into the remaining stump of the ascending aorta. The orifice and origin of the left coronary artery were identified above the left aortic cusp, and a Ch 6 infant feeding tube (Unomedical-ConvaTec limited, Deeside, UK) was gently inserted into the ostium to trace the short stem of the artery. To ensure that the catheter was maintained in place inside the anterior interventricular artery (AIA), careful dissection and isolation of the proximal $2 \mathrm{~cm}$ of the AIA was performed to enable its ligature with a $0.5-\mathrm{cm}$ wide satin band and an orthodontic rubber band (Fig. 1).

The exact same procedure was performed for the circumflex branch of the left coronary artery and for the right coronary artery.

\section{Thermal camera}

Thermal images were acquired through a FlirOne thermal camera (FLIR Systems ${ }^{\circledR}$, Wilsonville, Oregon, USA) paired with a Google Android Marsmallow 6.0 Smartphone with the FlirOne ${ }^{\mathrm{TM}}$ App (FLIR Systems, Wilsonville, Oregon, USA) installed.

\section{Preparation of contrast medium}

Three contrast media were prepared: contrast medium 1, consisting of ultrasound gel (CM1); contrast medium 2, consisting of a mix of ultrasound gel $(50 \%)$ and distilled water (50\%) (CM2); and contrast medium 3 consisting of distilled water (CM3).

\section{Thermal imaging coronarography}

After the cannulation of the AIA, each heart was placed in a refrigerated environment $\left(-20^{\circ} \mathrm{C}\right)$ for about $10 \mathrm{~min}$, until its temperature decreases to about $+4^{\circ} \mathrm{C}$. Ten millimeters of $\mathrm{CM}$ was heated to $40^{\circ} \mathrm{C}$, aspirated with a syringe and injected into the AIA through the Ch 6 infant feeding tube, while thermal images were captured through the thermal camera.
Fig. 1 a The satin band was looped under the previously isolated proximal $2 \mathrm{~cm}$ of the anterior interventricular artery (AIA). The two strings of the satin band were thus inserted into an orthodontic rubber band. b One of the two strings was passed again under the previously isolated proximal $2 \mathrm{~cm}$ of the AIA dragging the orthodontic rubber band under the AIA. Finally, the two strings were knotted

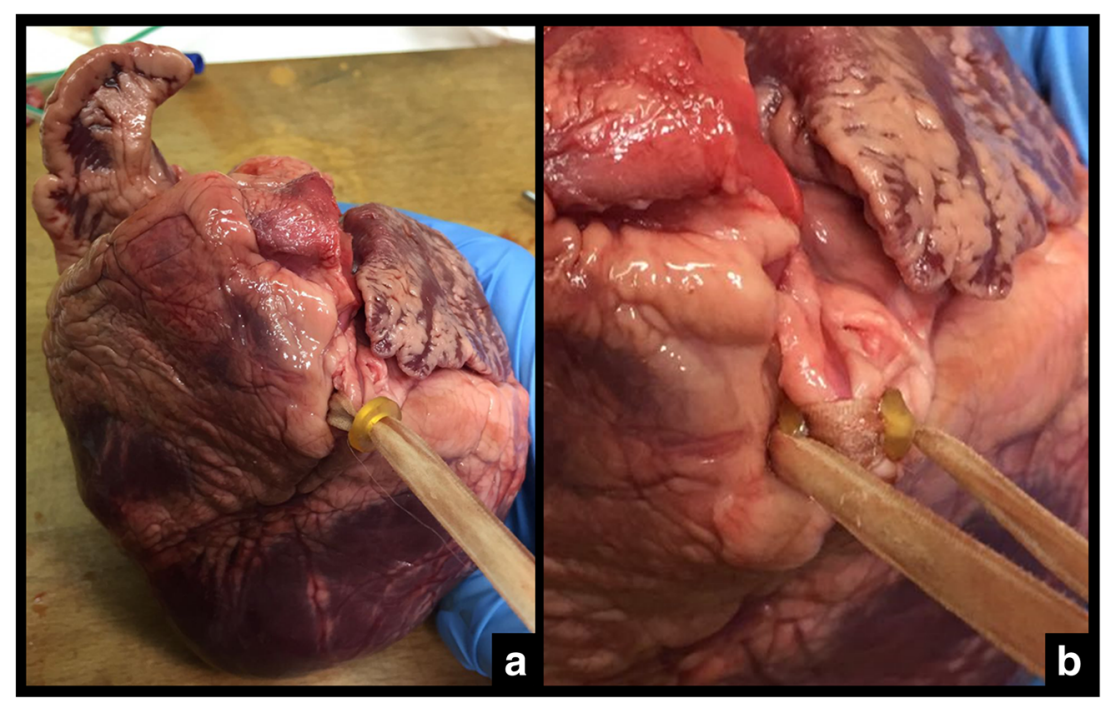


Table 1 Number of injections of different contrast media on each tested heart along the "selection of the contrast medium" phase of the study and the "thermal imaging coronarography under simulated pathologic conditions" phase of the study

\begin{tabular}{|c|c|c|c|c|c|c|c|c|}
\hline & \multicolumn{8}{|c|}{ Number of injections } \\
\hline & \multicolumn{2}{|l|}{ AIA } & \multicolumn{3}{|l|}{$\mathrm{CA}$} & \multicolumn{3}{|l|}{ RA } \\
\hline & $n H$ & $n R$ & $n H$ & & $n R$ & $n H$ & & $n R$ \\
\hline \multirow[t]{2}{*}{ CM1 } & $x$ & 5 & 5 & $\times$ & 5 & 5 & $\times$ & 5 \\
\hline & \multicolumn{2}{|l|}{ Tot. 25} & \multicolumn{3}{|c|}{ Tot. 25} & \multicolumn{3}{|c|}{ Tot. 25} \\
\hline \multirow[t]{2}{*}{$\mathrm{CM} 2$} & $x$ & 5 & 5 & $\times$ & 5 & 5 & $x$ & 5 \\
\hline & \multicolumn{2}{|l|}{ Tot. 25} & \multicolumn{3}{|c|}{ Tot. 25} & \multicolumn{3}{|c|}{ Tot. 25} \\
\hline \multirow[t]{3}{*}{ CM3 } & 5 & 5 & 5 & $\times$ & 5 & 5 & $\times$ & 5 \\
\hline & 15 & 4 & 15 & $\times$ & 4 & 15 & $\times$ & 4 \\
\hline & \multicolumn{2}{|l|}{ Tot. 85} & \multicolumn{3}{|c|}{ Tot. 85} & \multicolumn{3}{|c|}{ Tot. 85} \\
\hline \multirow[t]{2}{*}{ Occlusion } & & 5 & Not & rforr & & \multirow{2}{*}{\multicolumn{3}{|c|}{ Not performed }} \\
\hline & \multicolumn{5}{|l|}{ Tot. 50} & & & \\
\hline \multirow[t]{2}{*}{ Sub-occlusion } & $10 \times$ & 5 & Not & rforr & & \multirow{2}{*}{\multicolumn{3}{|c|}{ Not performed }} \\
\hline & \multicolumn{5}{|l|}{ Tot. 50} & & & \\
\hline
\end{tabular}

AIA anterior interventricular artery, $C A$ circumflex branch of the left coronary artery, $R A$ right coronary artery, $C M 1$ contrast medium $1, C M 2$ contrast medium 2, CM3 contrast medium $3, n H$ number of tested hearts, $n R$ number of repetitions of the injection

\section{Selection of the contrast medium}

The steps of the procedure previously described were performed for the AIA for the circumflex branch of the left coronary artery and for the right coronary artery of:

- Five hearts using CM1: the procedure was repeated five times for each tested coronary artery.

- Five hearts using CM2: the procedure was repeated five times for each tested coronary artery.

- Five hearts using CM3: the procedure was repeated five times for each tested coronary artery (Table 1).

For CM1 and CM2, prior to each injection, a further step, consisting of the washing out through abundant distilled water of the CM previously injected, was performed. The resulting thermal images were analyzed aiming at selecting the contrast medium more suitable to perform the further experimental trials.

TIC was performed for the AIA, for the circumflex branch of the left coronary artery and for the right coronary artery for the remaining 15 hearts, using CM3. These steps were repeated 60 times, for each coronary artery, namely 4 times on the AIA, 4 times on the circumflex branch, and 4 times on the right coronary artery, of each of the 15 hearts tested with CM3 (Table 1).

\section{Thermal imaging coronarography under simulated pathologic conditions}

- Aiming at simulating a complete occlusion on 10 hearts previously tested with CM3, the AIA was occluded $2 \mathrm{~cm}$ distally to its origin through a knot performed with a surgical suture (Ethilon® Nylon Suture) (Fig. 2a). After the occlusion of the AIA, each heart was placed in a refrigerated environment $\left(-20^{\circ} \mathrm{C}\right)$ until its temperature decreases to about $+4{ }^{\circ} \mathrm{C}$. Then, the AIA was instilled with $10 \mathrm{~mL}$ of $\mathrm{CM} 3$ previously heated at $40{ }^{\circ} \mathrm{C}$ while thermal images were recorded. These steps were repeated 50 times, namely 5 times for the experimentally occluded AIA of each of the 10 tested hearts (Table 1).

- Aiming at simulating a sub-occlusion on the remaining 10 hearts previously tested with $\mathrm{CM} 3$, a small round-shaped plastic object presenting a minute hole (Fig. 2b) was introduced from the left coronary ostium and slowly pushed into the AIA. Then, each heart was placed in a refrigerated environment $\left(-20{ }^{\circ} \mathrm{C}\right)$ until its temperature decreases to about $+4{ }^{\circ} \mathrm{C}$ and the AIA was instilled with $10 \mathrm{~mL}$ of $\mathrm{CM} 3$ previously heated at $40^{\circ} \mathrm{C}$ while thermal images were recorded. These steps were repeated 50 times, namely 5 times for the sub-occluded AIA of each of the 10 tested hearts. The resulting thermal images were analyzed and compared to the corresponding images acquired on the AIA of each heart under normal conditions (Table 1).
Fig. 2 a Anterior interventricular artery (AIA) occlusion through a surgical suture. b Small roundshaped plastic object $($ diameter $=$ $0.6 \mathrm{~cm}$ ) presenting a minute hole $($ diameter $=0.2 \mathrm{~cm})$ which was pushed into the anterior interventricular artery (AIA) aiming at simulating a subocclusion

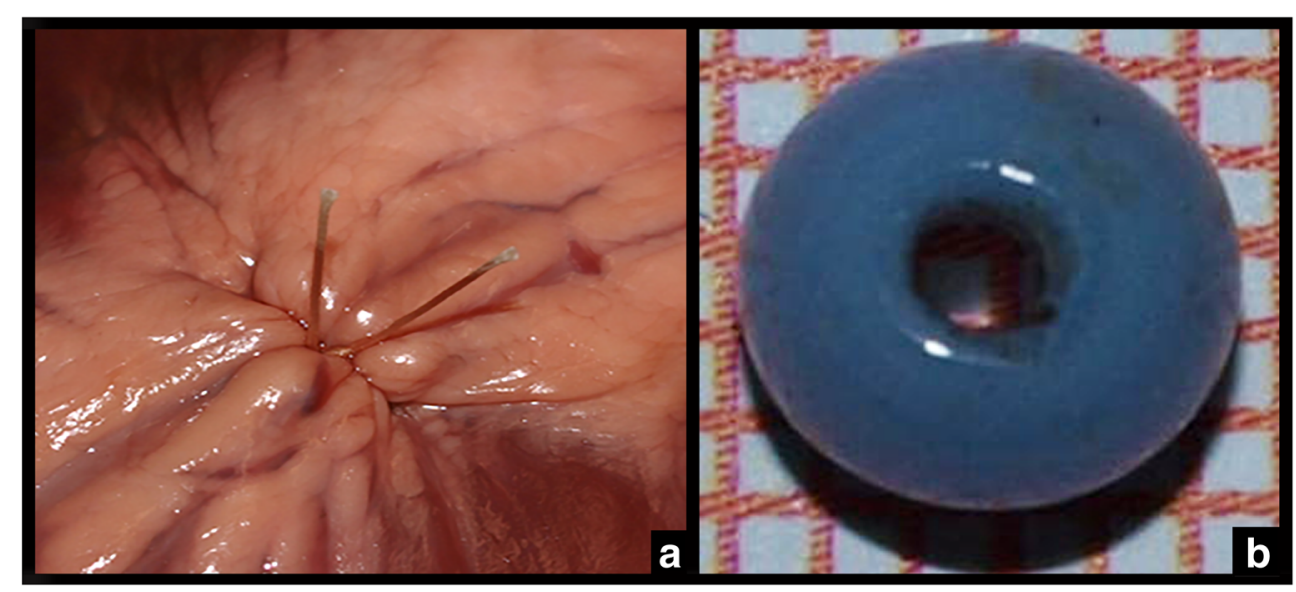




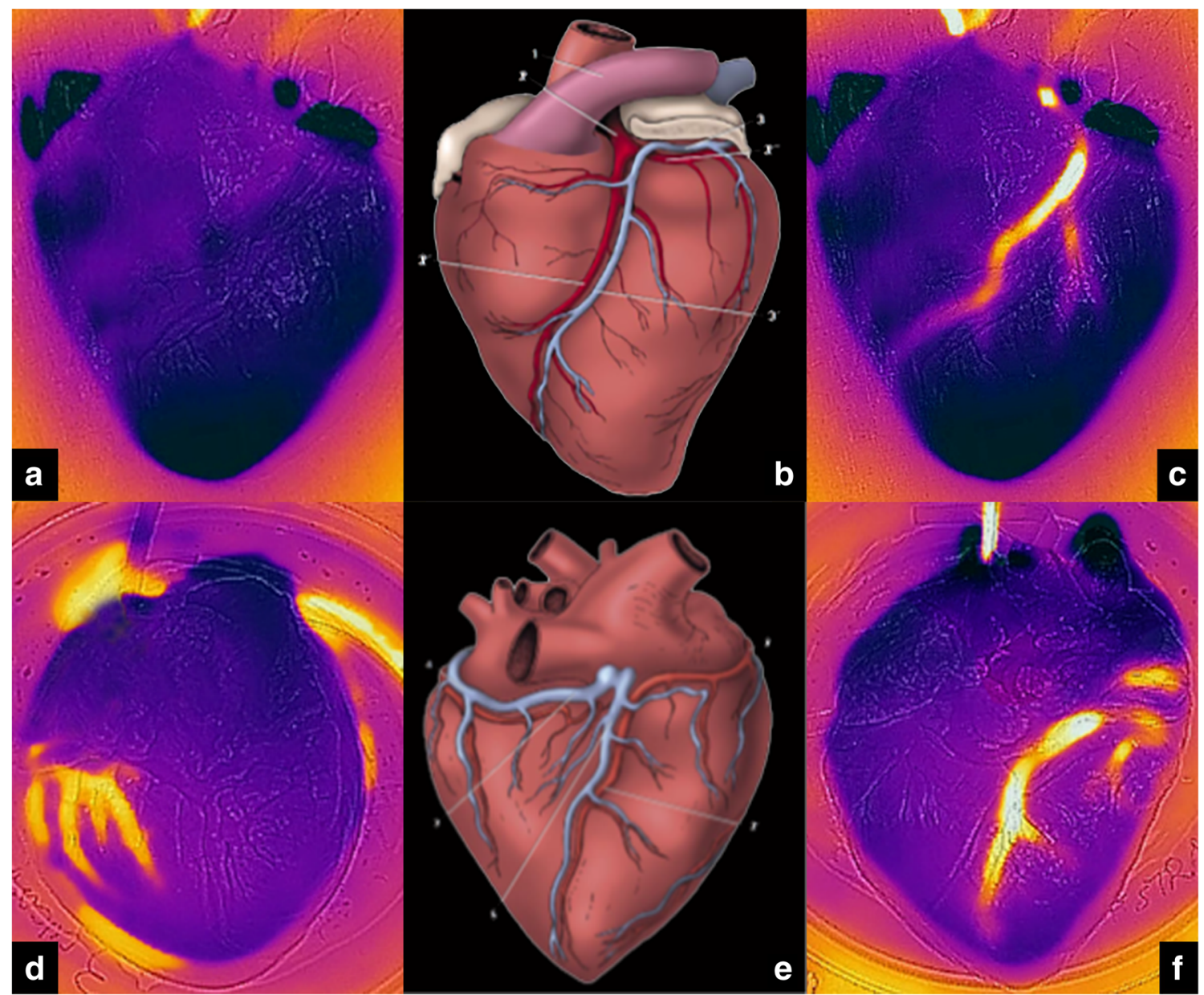

Fig. 3 Thermal imaging of the heart before the instillation of CM (a). Branching of the left coronary artery of the swine heart, adapted from the original Figs. 7-18 from Dyce K et al. [26] (b). TIC of the left coronary artery (c). TIC of the circumflex branch of left coronary artery (d).

\section{Examination of coronary arteries}

After TIC, the coronary vessels were examined making multiple transverse cuts at 3-mm intervals along the course of the main epicardial arteries, including branches such as the diagonal and obtuse marginal and checking patency.

Finally, on five swine hearts, after the instillation of $10 \mathrm{~mL}$ of CM3 into the AIA, the circumflex branch of the left coronary artery, and the right coronary artery, the myocardium was sampled according to guidelines for investigation of sudden cardiac death [26]. Myocardial samples were stained in hematoxylin and eosin, and histologic examination was performed.

\section{Results}

Our cannulation method, taking advantage of an elastic orthodontic rubber band, allowed a good perfusion of the coronary arteries in all the tested samples.
Branching of the circumflex branch of left coronary artery and of the interventricular branch of the right coronary artery of the swine heart adapted from the original Figs. 7-19 from Dyce K et al. [26] (e). TIC of the interventricular branch of the right coronary artery (f)

\section{Thermal imaging coronarography under normal conditions and selection of the CM}

After the cooling of the heart at $-20^{\circ} \mathrm{C}$ for $10 \mathrm{~min}$, the heart appeared uniformly colored in dark violet (Fig. 3a). After the instillation of $1-5 \mathrm{~mL}$ of $\mathrm{CM} 3$ the morphology and the course of the principal coronary vessels were clearly appreciable (Figs. 4a, $\mathrm{b}$ and $5 \mathrm{a}, \mathrm{b}$ ) while the thermal image of their secondary branches appeared only after the instillation of $5-10 \mathrm{~mL}$ of CM3 (Figs. 3c, d, f, 4c, d, and 5c, d). In particular, coronaries appeared colored in light violet, orange, yellow, and bright yellow varying along the course of the vessel and along the injection of CM3. All the investigated vessels resulted clearly distinguishable from the surrounding tissues, which were almost uniformly colored in dark violet (Fig. 3c, d, f).

Fig. 4 TIC of the AIA under normal conditions after the injection of $3 \mathrm{~mL}$ (a), $5 \mathrm{~mL}$ (b), $8 \mathrm{~mL}$ (c), and $10 \mathrm{~mL}$ (d) of CM3. TIC of the AIA of the same heart following complete occlusion (white arrow, $\mathbf{e}-\mathbf{h}$ ), after the injection of $3 \mathrm{~mL}(\mathbf{e}), 5 \mathrm{~mL}$ (f), $8 \mathrm{~mL}(\mathbf{g})$, and $10 \mathrm{~mL}$ (h) of CM3 


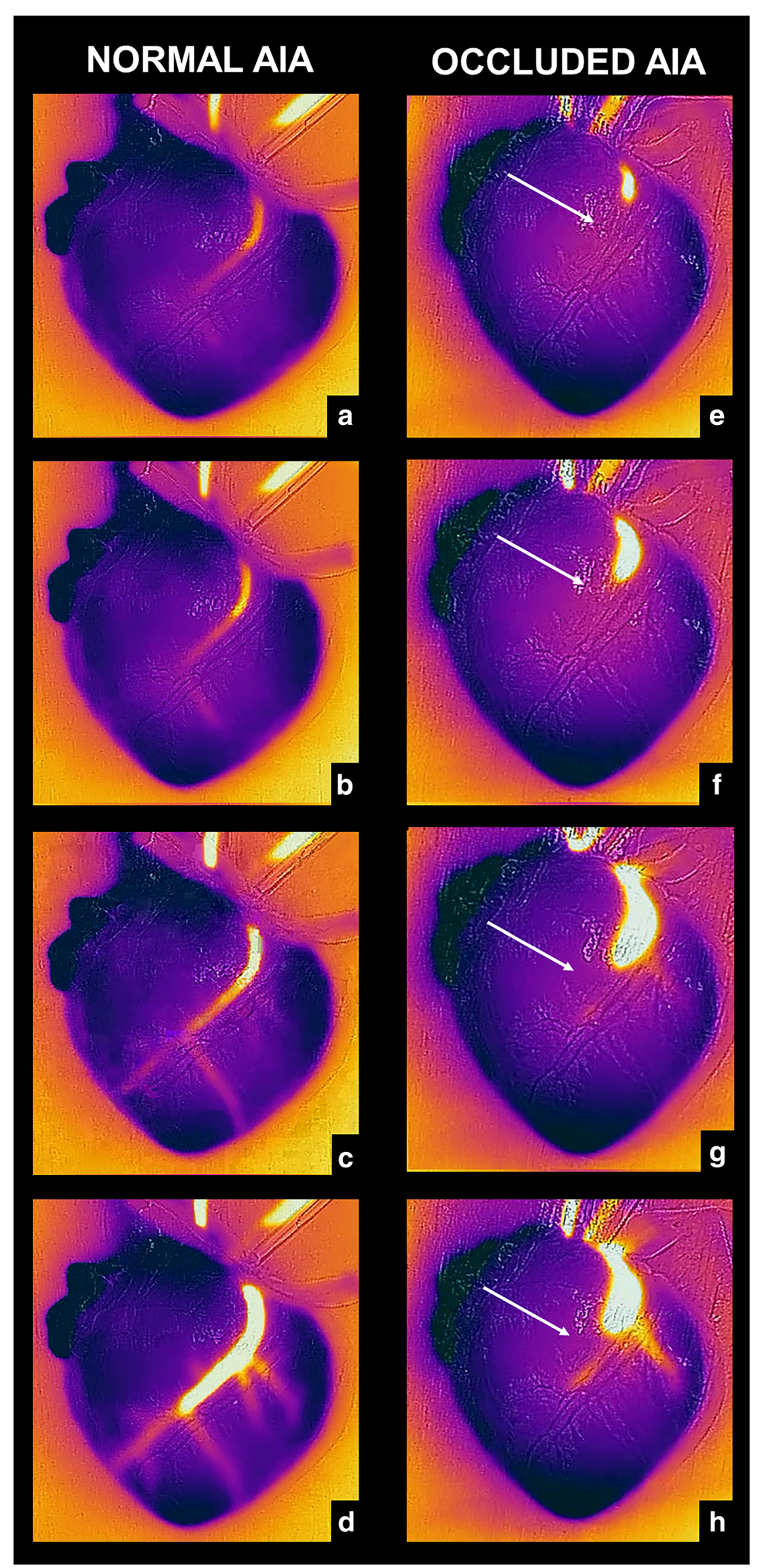




\section{NORMAL AIA}
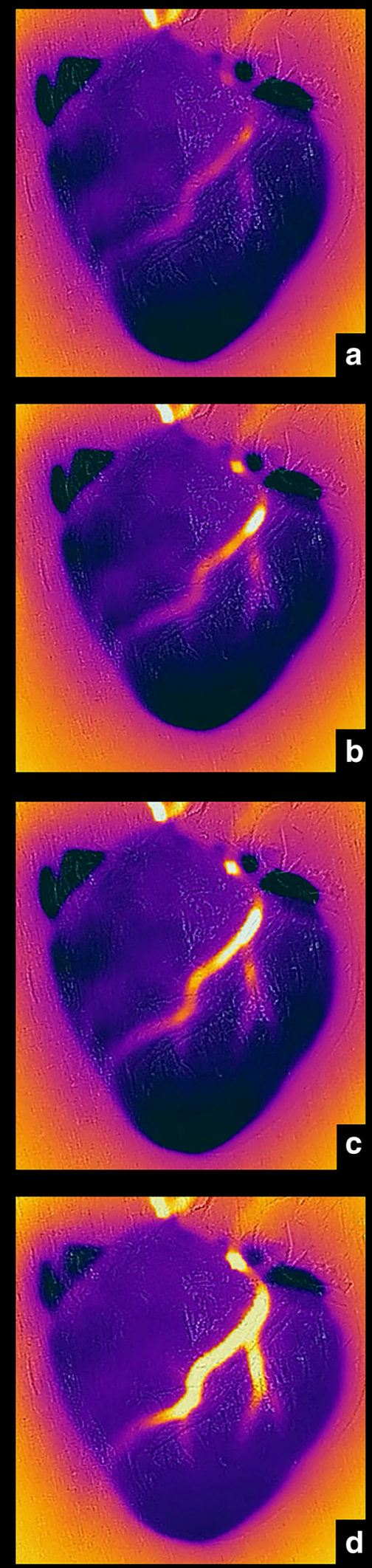

\section{AIA SUB-OCCLUSION}
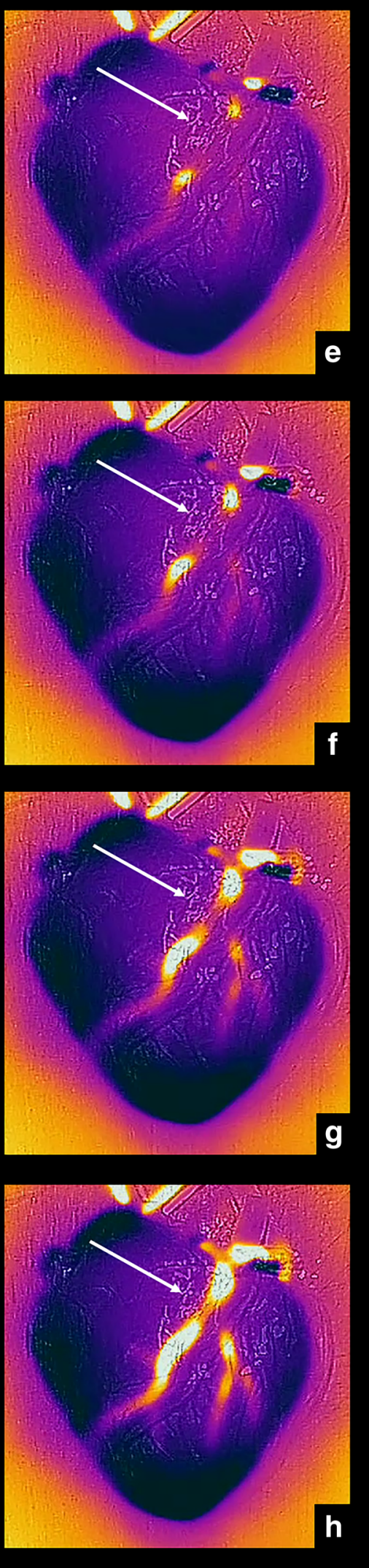
On the other hand, the secondary coronary branches of the AIA, the circumflex branch, and the right coronary artery appeared not perfused in the five hearts injected with CM1 and less perfused in the five hearts injected with CM2 (Fig. 6). Similar results were obtained when the procedure was repeated for each single vessel. Moreover, CM1 and CM2 needed to be washed out from the vessel through abundant instillation of distilled water before repeating the TIC, while this further step was unnecessary after the instillation of CM3. Basing on these results, CM3 was selected for the further trials, namely the TIC performed on the AIA, the circumflex coronary branch, and the right coronary artery on the remaining 15 hearts tested under normal conditions.

After the instillation of $\mathrm{CM} 3$, the course of the AIA was clearly depicted in all the tested samples (Figs. 4a-d and 5ad). However, along the course of both the right coronary artery (Figs. $3 \mathrm{f}$ and $7 \mathrm{a}$ ) and the circumflex artery (Figs. 3d and 7b), some isolated attenuation of the thermal signal, resulting in a focally light violet color, occurred when the vessel deepened into the epicardial fat and the myocardium. Similar results were obtained when the procedure was repeated for each single vessel.

\section{Thermal imaging coronarography under simulated pathologic conditions}

On 10 hearts previously injected through CM3, TIC was repeated after the complete occlusion of the vessel through a surgical suture (Fig. 2a). A clear demise of the thermal signal occurred after the occlusion (Fig. 4e-h, white arrow), while a distinct enhancement of the thermal signal was significant in the AIA segment proximal to the occlusion, especially after the injection of more than $5 \mathrm{~mL}$ of CM (Fig. $4 \mathrm{f}-\mathrm{h}$ ). Moreover, when the injection of $10 \mathrm{~mL}$ was completed, collateral secondary coronary branches were clearly appreciable at TI (Fig. 4h). Similar results were obtained when the procedure was repeated five times for each AIA under the same simulated pathological conditions.

On the remaining 10 hearts previously injected through CM3, TIC was repeated after the introduction of a small round-shaped plastic object (Fig. 2b) into the AIA. A clear demise of the thermal signal corresponding to the position of foreign object (Fig. 5e-h, white arrow) occurred while a distinct enhancement of the thermal signal was appreciable in the AIA segments both proximal and distal to the sub-occlusion (Fig. 5e-h). Similar results were obtained when the procedure was repeated for each AIA under the same simulated pathological conditions.

Fig. 5 TIC of the AIA under normal conditions after the injection of $3 \mathrm{~mL}$ (a), $5 \mathrm{~mL}$ (b), $8 \mathrm{~mL}$ (c), and $10 \mathrm{~mL}$ (d) of CM3. TIC of the AIA of the same heart following sub-occlusion (white arrow, $\mathbf{e}-\mathbf{h}$ ), after the injection of $3 \mathrm{~mL}(\mathbf{e}), 5 \mathrm{~mL}$ (f), $8 \mathrm{~mL}$ (g), and $10 \mathrm{~mL}$ (h) of CM3

\section{Examination of coronary arteries}

The gross examination of coronary vessels, performed through multiple transverse cuts at 3-mm intervals, excluded the presence of atherosclerotic disease. Moreover, very short tracts of both the right and the circumflex artery deepen into the epicardial tissues being covered from fat and myocardium. At histopathological examination of the myocardium after the instillation of distilled water, alterations were not observed (Fig. 8).

\section{Discussion}

TI allows the detection of infrared radiation, which is a function of temperature, and produces thermal images displaying temperature differences of the studied object. In fact, the available TI studies of the cardiovascular system are not strictly categorizable as angiographic imaging studies because they were aimed at depicting the vascularization of a body district rather than a single vessel. Indeed, mammals are homeotherm and in particular cases different body districts may display physiologic and/or pathologic slight differences of temperature [3-19]. However, a significant difference of temperature between tissues and the circulating blood suitable to image through TI single vessels with an adequate degree of accuracy is not presumable in a living mammal. On the other hand, after the decease, the body cools and it is usually preserved in a cool or refrigerated environment before autopsy to slow down postmortem alterations. Basing on this key concept, a significant difference in temperature between a vessel injected with a hot $\mathrm{CM}$ and the surrounding tissues, furthermore if previously cooled, may be expected at post-mortem TI. On these grounds, angiographic applications of TI to analyze single vessels in a post-mortem setting seemed plausible and thus TI was tested in this pilot study on coronary arteries of ex situ hearts. Swine hearts were selected to test TIC because the coronary arteries of the pig are very similar to that of humans [27-29]. The results of this study are herein analytically discussed.

\section{Selection of the CM for TIC}

If compared to the instillation of CM2 and CM1, the injection of CM3 allowed the best depiction of the morphology and the course of coronary vessels, permitting to observe not only the principal coronary vessels but also their secondary branches. These results are probably related to the lower density of CM3 (100\% distilled water) with respect to CM2 (50\% of ultrasound gel and $50 \%$ of distilled water) and CM1 (100\% ultrasound gel). Indeed, the lower density of CM3 probably allowed a better perfusion of small caliber secondary vessels, which was difficult with $\mathrm{CM} 2$ and hindered with $\mathrm{CM} 1$. Moreover, CM1 and CM2 needed to be washed out through abundant instillation of distilled water before repeating the 


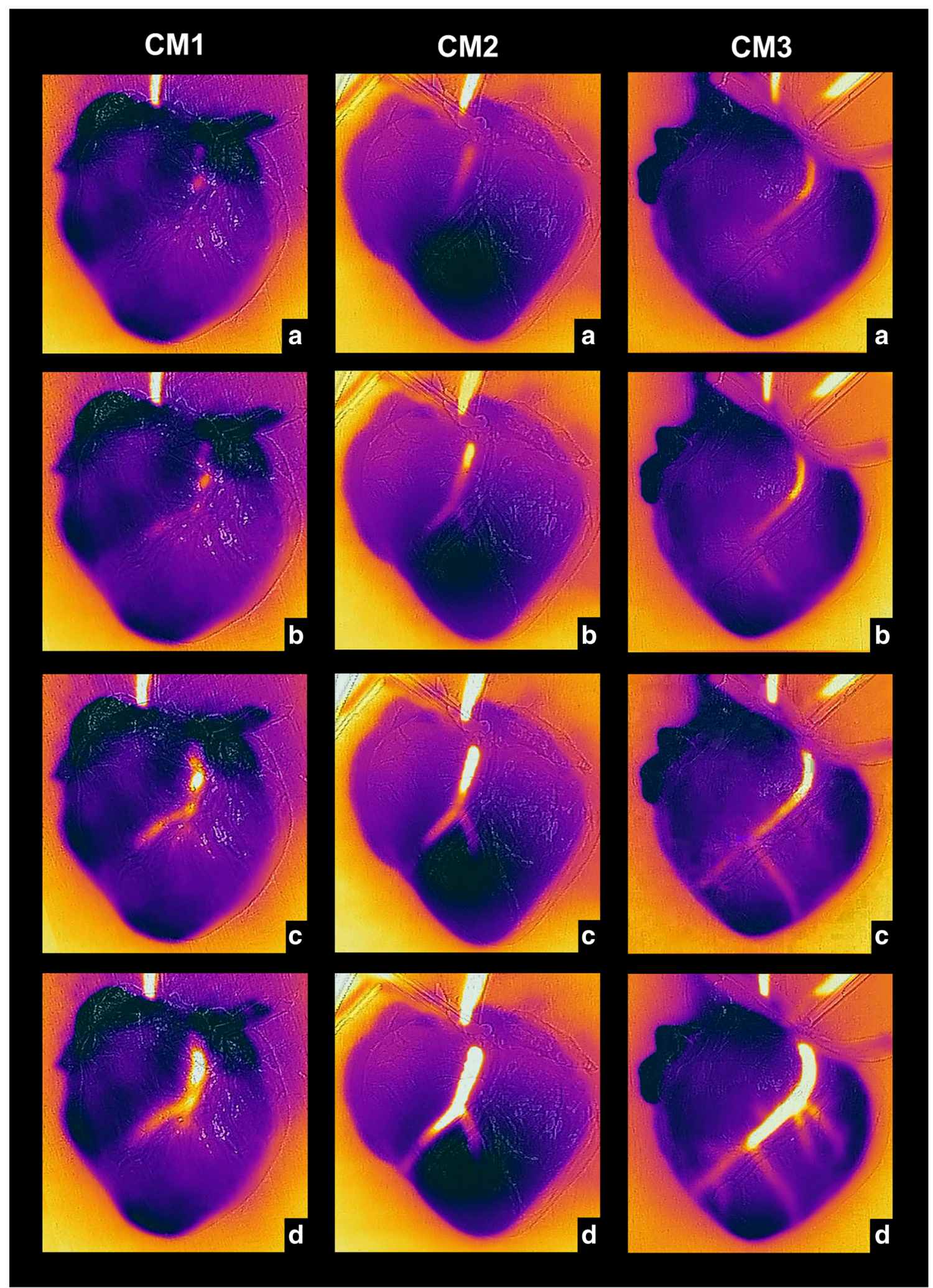

Fig. 6 TIC of the AIA under normal conditions after the injection of $3 \mathrm{~mL}$ (a), $5 \mathrm{~mL}$ (b), $8 \mathrm{~mL}$ (c), and $10 \mathrm{~mL}$ (d) of CM. After the injection of CM1 (first column), the course of the principal coronary vessels was appreciable. After the injection of CM2 (second column), the course of the principal coronary vessels was appreciable and the secondary coronary branches were partially appreciable. After the injection of CM3 (third column), the morphology and the course of the principal coronary vessels and of their secondary branches were clearly appreciable 
Fig. 7 a TIC of the right coronary artery, some isolated attenuation of the thermal signal, resulting in a focally light violet color, occurred when the vessel deepened into the epicardial fat and the myocardium. b TIC of the circumflex branch of the left coronary artery (left lateral view of the heart), some isolated attenuation of the thermal signal, resulting in a focally light violet color, occurred when the vessel deepened into the epicardial fat and the myocardium

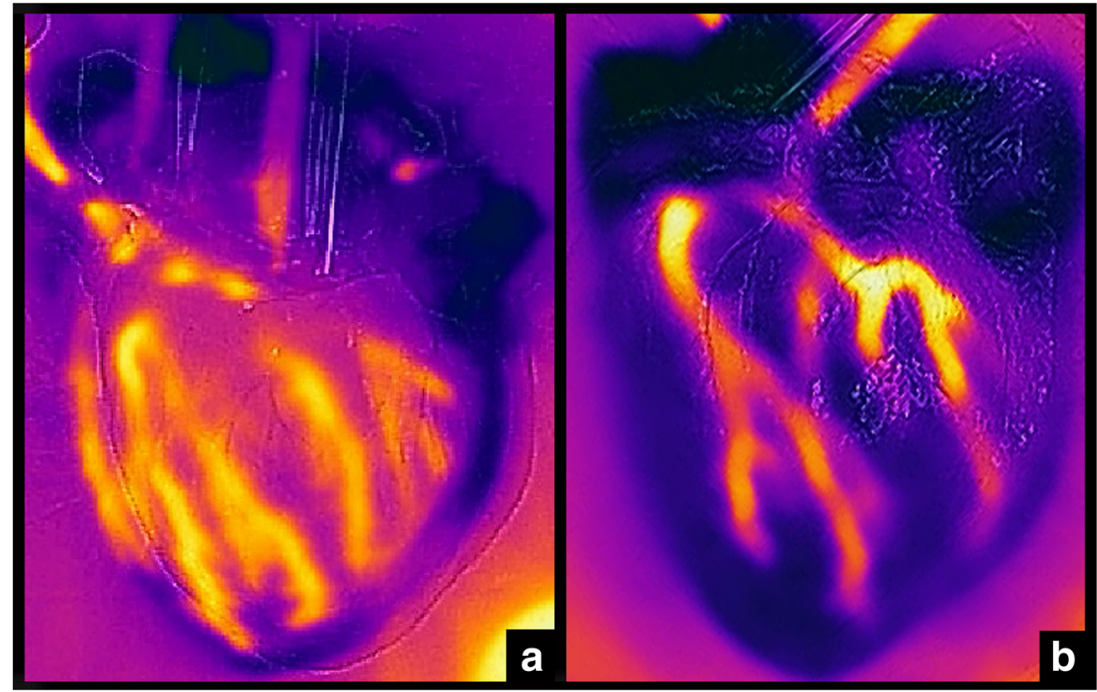

injection, while this further step was unnecessary after the instillation of CM3; thus, CM3 was selected for TI of further 15 hearts and for the additional trials performed on the experimentally occluded or sub-occluded AIA.

Although further studies are necessary to confirm our results on hearts presenting real coronary heart disease, we can hypothesize several advantages related to the use of CM3. Considering future possible applications of TIC on human hearts, the injection of a CM denser than blood may entail a

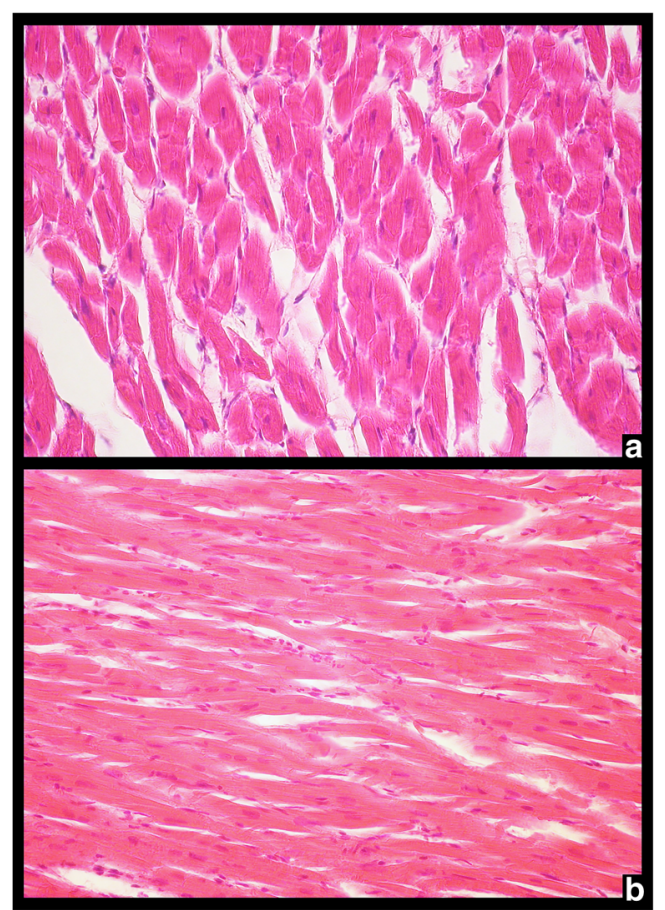

Fig. 8 Histopathological images of myocardial tissue sampled in the boundaries of the AIA before (a) and after (b) TIC. Alteration of the myocardial tissues were not observed after the injection of distilled water at $40{ }^{\circ} \mathrm{C}(\mathrm{CM} 3)$ mechanical stress suitable to alter intra-vitam pathological conditions, for example, breaking the adhesion between a thrombus and a coronary artery plaque and thus displacing a thrombosis. On the other hand, there is no apparent reason to hypothesize the alteration of intra-coronary pathological conditions when a CM less dense than blood, such as CM3, is injected into a coronary. Moreover, the injection of $\mathrm{CM}$ with a temperature $\left(40^{\circ} \mathrm{C}\right)$ similar to blood in a living person probably avoids also thermal stress. Finally, no histopathological alterations related were observed at histopathological examination after the injection of distilled water.

\section{Thermal imaging coronarography under normal conditions}

TIC allowed to clearly delineate all the investigated coronary arteries, which were colored in light violet, orange, yellow, and bright yellow, and to distinguish them from the surrounding myocardial tissue, which was almost uniformly colored in dark violet (Fig. 3). TI is based on the conversion of invisible infrared radiation into visible images to visualize temperature differences. In particular, the contrast between myocardial tissue, which was previously cooled for $10 \mathrm{~min}$ at $-20^{\circ} \mathrm{C}$, and the coronary arteries, which were injected with $\mathrm{CM} 3$ at $40{ }^{\circ} \mathrm{C}$, was suitable to appreciate the morphology and course of the main coronary vessels. The chromatic variation along the course of coronary arteries is a function of the temperature of the liquid inside the vessel. As demonstrated from our results along the course of the coronary artery, the CM3 releases heath to the surrounding tissues, being hotter (bright yellow) proximally and gradually lowering its temperature distally, becoming yellow, orange, and finally light violet. The coloration of the coronary artery is of course also a function of the amount of the injected contrast medium. Indeed, as appreciable in the later phases of injection, the $5-10 \mathrm{~mL}$ of CM3 were 
capable to heat a more prolonged segment of coronary artery (Figs. 4c, d and 5c, d).

TIC of the AIA allowed a clear depiction of the vessel in all the tested samples (Figs. 4a-d and 5a-d). On the other hand, along the course of both the right coronary artery (Figs. $3 \mathrm{f}$ and 7a) and the circumflex branch of the left coronary artery (Figs. $3 \mathrm{~d}$ and $7 \mathrm{~b}$ ), some isolated attenuation of the thermal signal, resulting focally light violet in color, occurred when the vessel deepened into the epicardial fat and the myocardium, which thermally insulate the coronary vessel. After TIC trials, results were confirmed at gross examination, performed through multiple transverse cuts of coronary arteries. The further experimental trials simulating pathological conditions were performed on the AIA, because of the more uniform thermal signal deriving from the TIC performed on this vessel.

\section{Thermal imaging coronarography under simulated pathologic conditions}

Sudden cardiac death (SCD) is defined as a natural, unexpected fatal event due to cardiovascular collapse occurring within 1 hour since the onset of initial symptoms [30]. The most frequent cause of SCD in the general population in industrialized countries is atherosclerotic disease and, in particular, coronary atherosclerosis, which leads to impaired cardiac blood supply. Basing on these general concepts and in the absence of cardiopathological conditions in the swine tested sample, in the present study, we chose to experimentally simulate a total obstruction and a sub-obstruction of the AIA to test a possible role of TIC in the post-mortem diagnosis of coronary heart disease on ex situ hearts. In particular, total occlusion and subocclusion were selected for these exploratory tests because of their important hemodynamic effects, which were presumed to be more clearly appreciable through TIC. The occluding plaque was simulated through the surgical suture of the AIA because it was expected to totally occlude the blood supply after the occlusion. On the other hand, the sub-occluding plaque was simulated through the insertion of a foreign body into the AIA. A round-shaped foreign body was chosen because the coronary vessel is round-shaped too; thus, a good adhesion with the vessel walls could be expected. The diameter of the foreign object, slightly lower than the left coronary ostium, allowed to push it into the coronary artery for some centimeters until the progression was blocked by the consensual distal decrease of the caliber of the AIA. Finally, the $0.2-\mathrm{cm}$ hole in the center of the foreign body was expected to allow the residual passage of $\mathrm{CM} 3$, simulating the hemodynamic setting occurring along a suboccluding atherosclerotic plaque.

After the occlusion of the AIA, the complete demise of the thermal signal (Fig. $4 \mathrm{e}-\mathrm{h}$ ) was clearly related to the ceasing of the downstream circulation. On the other hand, the distinct enhancement of the thermal signal in the AIA segment proximal to the occlusion, displaying a bright yellow signal, appeared mainly related to the backward accumulation of hot contrast medium (Fig. 4f-h). Finally, after the injection of $10 \mathrm{~mL}$ was completed, two collateral secondary AIA branches were clearly appreciable at TI (Fig. 4h), suggesting a possible role of TIC also for the assessment of collateral compensatory circulation, often occurring in cases of chronic coronary heart disease.

Considering thermal images acquired following experimental simulated sub-occlusion of the AIA, the clear demise of thermal signal corresponding to the foreign object was associated to an enhancement of the coronary artery thermal signal both proximal and distal to the sub-occlusion (Fig. $5 \mathrm{e}-\mathrm{h})$. This thermographic pattern appears to be markedly different from the demise of signal related to the deepening of the vessel into the epicardial fat and the myocardium (Fig. $3 \mathrm{~d}, \mathrm{f}$ ). On these grounds, under our experimental conditions, the differential diagnosis between these conditions through TIC resulted possible and quite simple.

\section{Conclusions}

Post-mortem TI allowed, under our experimental conditions, the analysis of single coronary vessels and to distinguish the vascular structures from the surrounding tissues. Indeed, a proper difference of temperature between vessels, injected with hot $\mathrm{CM}$, and the surrounding tissues (i.e., the background) was achieved because of post-mortem cooling, which was aided through the cooling of the studied animal sample. Thus, the results of this preliminary pilot investigation, performed on swine coronary arteries, seem encouraging to test further angiographic applications of TI in human post-mortem samples.

Basing on our preliminary results, TIC, allowing a clear and reproducible depiction of coronary vessels and of simulated coronary heart disease, might be proposed as a technique allowing subsequent further imaging studies [25, 31-35], indeed, especially if performed together with MPMCTA can not only orient about the cause of death before autopsy but can also help to identify affected regions for guiding and improving the sampling for microscopic examination [36]. In conclusion, these techniques may help to achieve a more focused cardiopathological examination of the heart, which remains mandatory for the definitive diagnosis of coronary heart disease [26]. The cannulation of coronary vessels is easy to perform; the thermal camera and the related app interface are user-friendly, cheap, and portable. Moreover, the catheter, the $\mathrm{CM}$ (hot distilled water), and a freezer at $-20^{\circ} \mathrm{C}$ are available almost everywhere. Considering these undeniable advantages, TIC might be performed during the autopsy or even later and in a different setting on the ex situ heart. Finally, TIC is non-destructive and might be repeated virtually infinite times on the same sample. Obviously, further systematic studies on human samples are necessary to draw any definitive conclusion. 


\section{Compliance with ethical standards}

Conflict of interest The authors declare that they have no conflict of interest.

\section{References}

1. Ammer K, Ring EFJ (2005) Influence of the field of view on temperature readings from thermal images. Thermol Int 15:99-103

2. Kateb B, Yamamoto V, Yu C, Grundfest W, Gruen JP (2009) Infrared thermal imaging: a review of the literature and case report. Neuroimage 47:154-162. https://doi.org/10.1016/j.neuroimage. 2009.03.043

3. Love TJ (1980) Thermography as an indicator of blood perfusion. Ann N Y Acad Sci 335:429-437

4. Merla A, Romani GL (2006) Functional infrared imaging in clinical applications. In: Bronzino JD (ed) The biomedical engineering handbook. CRC Press, USA, pp 32.1-32.16

5. Keyserlingk JR, Ahlgren PD, Yu E, Belliveau N, Yassa M (2000) Functional infrared imaging of the breast. IEEE Eng Med Biol Mag 19:30-41

6. Head JF, Lipari CA, Elliott RL (1999) Comparison of mammography and breast infrared imaging: sensitivity, specificity, false negatives, false positives, positive predictive value and negative predictive value. 21st Ann Int Conf IEEE Eng Med Biol Soc. https://doi. org/10.1109/IEMBS.1999.804278

7. Gautherie M, Gros CM (1980) Breast thermography and cancer risk prediction. Cancer 45:51-56

8. Stark AM (1985) The value of risk factors in screening for breast cancer. Eur J Surg Oncol 11:147-150

9. Cole RP, Jones SG, Shakespeare PG (1990) Thermographic assessment of hand burns. Burns 16:60-63

10. Hargroder AG, Davidson JE, Luther DG, Head JF (1999) Infrared imaging of burn wounds to determine burn depth. Infrared Tech Applic 3698:103-108. https://doi.org/10.1117/12.354509

11. Esselinckx W, Bacon PA, Ring EF, Crooke D, Collins AJ, Demottaz D (1978) A thermographic assessment of three intra-articular prednisolone analogues given in rheumatoid synovitis. Br J Clin Pharmacol 5:447-451

12. Bird HA, Ring EF, Bacon PA (1979) A thermographic and clinical comparison of three intra-articular steroid preparations in rheumatoid arthritis. Ann Rheum Dis 38:36-39

13. Bacon PA, Ring EFJ, Collins AJ (1977) Thermography in the assessment of anti rheumatic agents. In: Gordon JL, Hazleman BL (eds) Rheumatoid arthritis. Elsevier/North Holland Biomedical Press, Amsterdam, p 105

14. Fischer AA, Chang CH (1986) Temperature and pressure threshold measurements in trigger points. Thermology 1:212-215

15. Schmitt M, Guillot Y (1984) Thermography and muscular injuries in sports medicine. In: Ring EFJ, Phillips B (eds) Recent advances in medical thermology. Springer, Boston, pp 439-445

16. Ng EYK, Kawb GJL, Chang WM (2004) Analysis of IR thermal imager for mass blind fever screening. Microvasc Res 68:104-109

17. $\mathrm{Ng} \mathrm{EY} \mathrm{(2005)} \mathrm{Is} \mathrm{thermal} \mathrm{scanner} \mathrm{losing} \mathrm{its} \mathrm{bite} \mathrm{in} \mathrm{mass} \mathrm{screening} \mathrm{of}$ fever due to SARS? Med Phys 32:93-97

18. Chan LS, Cheung GT, Lauder IJ, Kumana CR, Lauder IJ (2004) Screening for fever by remote-sensing infrared thermographic camera. J Travel Med 11:273-279
19. Li WW, Head JF (2000) Infrared imaging in the detection and evaluation of tumor angiogenesis. 22nd Ann Int Conf IEEE Eng Med Biol Soc. https://doi.org/10.1109/IEMBS.2000.900470

20. Szabò Z, Berg S, Sjokvist S, Gustafsson T, Carleberg P, Uppsall M, Wren J, Ahn H, Smedby O (2013) Real-time intraoperative visualization of myocardial circulation using augmented reality temperature display. Int J Card Imaging 29:521-528

21. Mall G, Hubig M, Beier G, Buttner A, Eisenmenger W (2002) Supravital energy production in early post-mortem phase - estimate based on heat loss due to radiation and natural convection. Legal Med 4:71-78

22. Johnson AP, Mikac KM, Wallman JF (2013) Thermogenesis in decomposing carcasses. Forensic Sci Int 231:271-277. https://doi. org/10.1016/j.forsciint.2013.05.031

23. Desmarais AM (2014) Detection of cadaveric remains by thermal imaging cameras. J Forensic Ident 64:489-510

24. Amendt J, Rodner S, Schuch CP, Sprenger H, Weidlich L, Reckel F (2017) Helicopter thermal imaging for detecting insect infested cadavers. Sci Justice 57:366-372. https://doi.org/10.1016/j.scijus. 2017.04.008

25. Bruguier C, Egger C, Vallèe JP, Grimm J, Boulanger X, Jackowski C, Mangin P, Grabherr S (2015) Postmortem magnetic resonance imaging of the heart ex situ: development of technical protocols. Int J Legal Med 129:559-567

26. Basso C, Aguilera B, Banner J et al (2017) Guidelines for autopsy investigation of sudden cardiac death: 2017 update from the Association for European Cardiovascular Pathology. Virchows Arch 471:691-705

27. Dyce K, Sack W, Wensing CJG (2010) Textbook of veterinary anatomy. Saunders-Elsevier, St. Louis, pp 233-234

28. Weaver ME, Pantely GA, Bristow JD, Ladley HD (1986) A quantitative study of the anatomy and distribution of coronary arteries in swine in comparison with other animals and man. Cardiovasc Res 20:907-917

29. Mokrane FZ, Dercle L, Meyrignac O, Crubezy E, Rousseau H, Telmon N, Dedouit F (2018) Towards multi-phase postmortem CT angiography in children: a study on a porcine model. Int J Legal Med 132:1391-1403. https://doi.org/10.1007/s00414-018-1783-y

30. Goldstein S (1982) The necessity of a uniform definition of sudden coronary death: witnessed death within 1 hour of the onset of acute symptoms. Am Heart J 103:156-159

31. Michaud K, Grabherr S, Doenz F, Mangin P (2012) Evaluation of postmortem MDCT and MDCT-angiography for the investigation of sudden cardiac death related to atherosclerotic coronary artery disease. Int J Card Imaging 28:1807-1822

32. Palmiere C, Lobrinus JA, Mangin P, Grabherr S (2013) Detection of coronary thrombosis after multi-phase postmortem CT-angiography. Leg Med (Tokyo) 15:12-18

33. Jackowski C, Schwendener N, Grabherr S, Persson A (2013) Postmortem cardiac 3-T magnetic resonance imaging: visualization of sudden cardiac death? J Am Coll Cardiol 62:617-629

34. Michaud K, Grabherr S, Jackowski C, Bollmann MD, Doenz F, Mangin P (2014) Postmortem imaging of sudden cardiac death. Int J Legal Med 128:127-137

35. Michaud K, Grabherr S, Faouzi M, Grimm J, Doenz F, Mangin P (2015) Pathomorphological and CT-angiographical characteristics of coronary atherosclerotic plaques in cases of sudden cardiac death. Int J Legal Med 129:1067-1077

36. Sabatasso S, Vanhaebost J, Doenz F, Palmiere C, Michaud K, Dedouit F, Grabherr S (2018) Visualization of myocardial infarction in postmortem multiphase computed tomography angiography: a feasibility study. Am J Forensic Med Pathol 39:106-113 